\title{
Bundle Adjustment for Marker-Based Rigid MR/X-Ray Registration
}

\author{
Peter Fischer, Member, IEEE, Thomas Pohl, Alexander Brost, Joachim Hornegger, Member, IEEE
}

\begin{abstract}
Rigid registration is a key requirement to overlay anatomical structures from pre-operative magnetic resonance (MR) images onto live X-ray fluoroscopic images. Clinical applications are for instance radio frequency catheter ablation and transcatheter endomyocardial injection. We present a method for fully automatic marker-based detection and registration. The algorithm locates the markers in the MR volumetric data set automatically by combining homomorphic unsharp masking, adaptive thresholding, region growing, and shape analysis. In the X-ray images, the markers are localized in several 2-D images and then their 3-D positions are reconstructed. The 2-D X-ray localization is performed using adaptive thresholding and template matching. The 3-D X-ray marker positions are established using backprojection. Rigid registration between the two 3-D point sets is performed by an iterative closest point algorithm. The 3-D registration error using a flat panel detector $\mathrm{X}$-ray system is on average $1.5 \pm 1.8 \mathrm{~mm}$. The novelty of our approach is the incorporation of bundle adjustment to reduce the 2-D overlay errors. Bundle adjustment reduces the 2-D X-ray marker localization error from $0.6 \pm 0.8 \mathrm{~mm}$ to $0.4 \pm 0.5 \mathrm{~mm}$. The target registration error of our proposed method evaluated on eight datasets is on average $1.4 \pm 3.5 \mathrm{~mm}$. These targets were not used during registration. Accordingly, anatomical structures from MR are positioned more accurately.
\end{abstract}

\section{INTRODUCTION}

A GENERAL problem of displaying overlay images in Xray projections is the registration between the volumetric data set and the X-ray projections. This paper only deals with the computation of a rigid transformation between the two coordinate systems. For an accurate overlay, additional tasks like motion compensation and non-rigid registration to account for deformations are required, but not considered in this work.

In general, procedures performed under X-ray fluoroscopy (XF) guidance can benefit from the overlay of pre-operatively acquired magnetic resonance (MR) or computed tomography (CT) volumes. A common procedure in electrophysiology is radio frequency ablation to treat atrial fibrillation, particularly if drug treatment becomes ineffective [1], [2], [3]. Overlay images can visualize anatomic details like pulmonary veins that would otherwise be poorly visible. Transcatheter endomyocardial injection delivers biological materials for therapeutic

Manuscript received November 4, 2012. This work was supported by Siemens AG, Healthcare Sector, Forchheim, Germany.

P. Fischer is with the Department of Computer Science, University Erlangen-Nuremberg, Erlangen, Germany (e-mail: peter.fischer@etechnik.stud.uni-erlangen.de).

T. Pohl is with Siemens Healthcare, Forchheim, Germany (e-mail: thomas.tp.pohl@siemens.com).

A. Brost is with the Department of Radiology, Stanford University, Stanford, CA 94305 USA (e-mail: brost@stanford.edu).

J. Hornegger is with the Department of Computer Science, University Erlangen-Nuremberg, Erlangen, Germany. (e-mail: jh@cs.fau.de). purposes [4]. There, exact device placement is important for high efficacy and minimal risk. When closing ventricular septal defects, X-ray fused with MR guidance can facilitate a different access route to the location of the defect, thus speeding up the procedure and reducing the radiation exposure of the patient [5].

During most procedures, registration is performed manually at the beginning of the procedure. This is difficult because the overlaid structure is poorly visible under X-ray. The task can be simplified in some cases, e.g. for cardiac applications by injecting contrast agent into the blood of the patient to enhance vessels, atria and ventricles. Overall, manual registration takes a considerable amount of time, which increases procedure time and bears additional risks. Therefore, automatic registration methods are investigated by the medical image processing community. One option is to acquire a C-arm CT after the patient is moved to the interventional suite [6]. Then a 3-D/3-D registration between the pre-operatively acquired volumetric data set and the C-arm CT data set can be performed. The disadvantage is that hundreds of image acquisitions are necessary to compute a $\mathrm{C}$-arm $\mathrm{CT}$ which requires additional dose for the patient. Rhode et al. use an optical tracking system that locates all the involved devices in the room using external optical markers [2]. This requires a separate tracking system, and movements of the patient relative to the table cannot be tracked. Tomazevic and Gueziec rely on anatomical landmarks [7], [8]. Gueziecs method requires the segmentation of bones in $\mathrm{CT}$ and of contours in the X-ray projection images. Inaccurate segmentations introduce additional errors during the registration process. It is also possible to use intensity-based similarity measures between the volumetric data set and the projections to find the best transformation [9], [10], [11].

Conceptually, this paper is based on the publication of George et al., who used external fiducial markers that are visible in both modalities to calculate the registration [12]. This approach has high accuracy and requires only a few projections. Thus, the radiation dose required for registration is low. In addition, movements of the patient relative to the table can be compensated for. The drawback is that the markers might also disturb the physician during the procedure by occluding important areas. This can be avoided by carefully choosing the marker positions and the projection angle during the procedure.

\section{Materials And Methods}

There are several tasks that need to be performed for automatically registering MR and XF images. First, the markers 

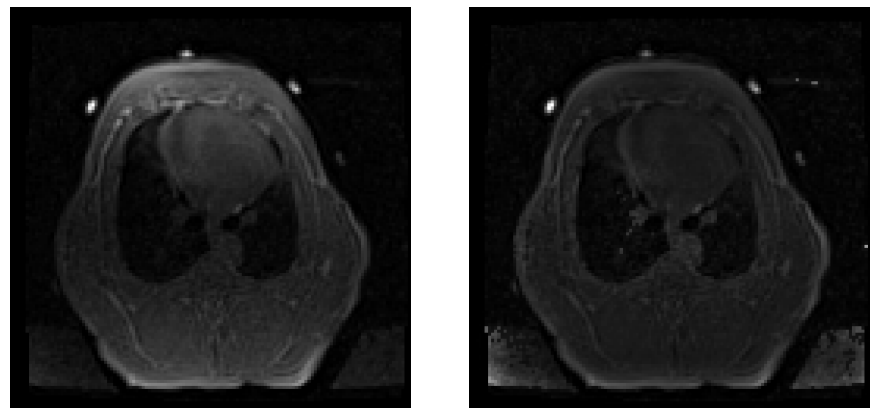

Fig. 1: An axial slice of an MR volume before (a) and after (b) bias field removal with homomorphic unsharp masking (HUM). The bright spots outside of the thorax are the markers.

have to be localized in both, MR and XF. Then, the 3-D positions of the XF markers must be reconstructed, which includes bundle adjustment. Finally, the rigid registration is computed using the 3-D point sets from MR and XF. All these steps are presented in this section.

\section{A. MR Marker Localization}

The markers are small tori filled with contrast agent with an outer diameter of $15 \mathrm{~mm}$, inner diameter of $5 \mathrm{~mm}$ and height of $5 \mathrm{~mm}$ (PinPoint; Beekley Corporation, Bristol, CT, USA). The contrast agent generates a response of high intensity in the MR images. The main features for localizing the markers are their high intensities, the high gradient between the markers and their surroundings, and the marker shape.

The use of intensities and gradients as features is disturbed by the MR intensity bias field. A simple and fast technique for removing the intensity bias field is homomorphic unsharp masking (HUM) [13]. An example is given in Fig. 1. The grey level is more uniform in the anatomy, which is particularly visible when comparing the top center and the middle left of the two images. Some artifacts are also introduced in the background, e.g. in the top right corner of the image.

In the bias-corrected images, candidate regions for the markers are extracted using gradient magnitude. The gradient is computed by convolving the image with the derivative of a Gaussian kernel. The gradient magnitude image is binarized using adaptive thresholding. This binary image is processed with morphological operations to fill small gaps inside the markers and to remove small isolated regions. Then, the remaining connected regions are identified. Each of the candidate regions is segmented based on intensity using simple region growing. The possibility of each region being a marker is assessed. Multiple tests based on prior knowledge about the markers have to be passed. The regions must have a 1.75 times higher intensity than their surrounding, and the maximum intensity must not be below 100. These values were found empirically. The shape of the regions is examined with principal component analysis. The 3-D positions of the region's voxels are considered as features. The explained variance of the three principal components has to be high for the first two and low for the third, which reflects the shape of a torus. In the end, the centroid of each region
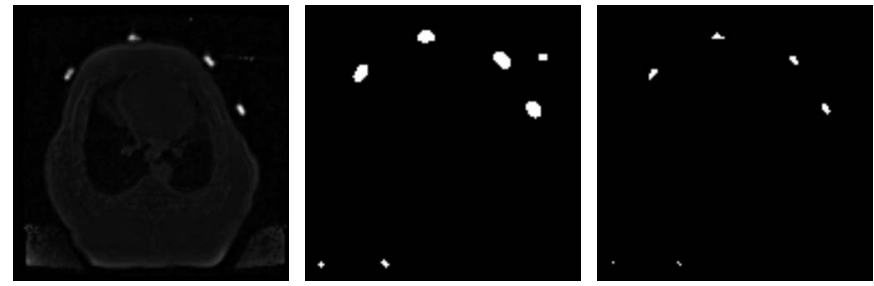

Fig. 2: The steps of marker localization illustrated on one axial slice of an MR volume.

is computed as the center of mass of the region, whereat voxel intensity is treated as mass. The remaining regions are sorted by maximum intensity in descending order. Fig. 2 demonstrates the progression of marker localization. Fig. 2(b) shows that all the markers are detected, along with a few false positives. Fig. 2(c) shows the same marker candidates, but the outlines of the candidates fit more closely to the true outlines, visible in Fig. 2(a). This is the basis for the shape analysis, which is able to reject the tiny candidates on the bottom of the image.

\section{B. X-Ray Marker Localization}

The accurate localization of the markers is a prerequisite for a good registration. There are special challenges in analyzing X-ray images caused by their fluoroscopic nature. First, the intensity of the markers depends on the background. If there is only air in the background, which has a lower attenuation coefficient than bones or other anatomical structures, the markers will appear brighter in the projection images compared to if anatomical structures are in the background of markers. Second, a single marker does not have a homogeneous intensity. Further difficulties arise from the perspective projection of the markers.

Markers are darker than their immediate surroundings assuming that the background is the same. This is caused by the higher attenuation coefficient of markers compared to air, which is surrounding the markers attached to the skin of the patients. An image processing technique that highlights relatively dark regions is the morphological operation of blackhat [14]. An elliptical structuring element of 1.5 times the size of the markers is used, as only regions that are smaller than the structuring element are highlighted by blackhat. The effects of blackhat on the X-ray fluoroscopy images can be seen in Fig. 3b.

The image is binarized with adaptive thresholding. The resulting binary image contains also false positives for several dark anatomical regions, like ribs. In addition, not every pixel of the markers is above the thresholds and the markers may be connected to anatomical regions. Actual marker detection is facilitated by template matching. The shape of a marker in 3-D is known, but the projections look different depending on the projection angle. Most markers appear similar to a disc with a hole, which is the first template shape. A second template is created for markers imaged under extreme projection angles resulting in two rectangles on top of each other. It is necessary to conduct the search with different template sizes, as the 

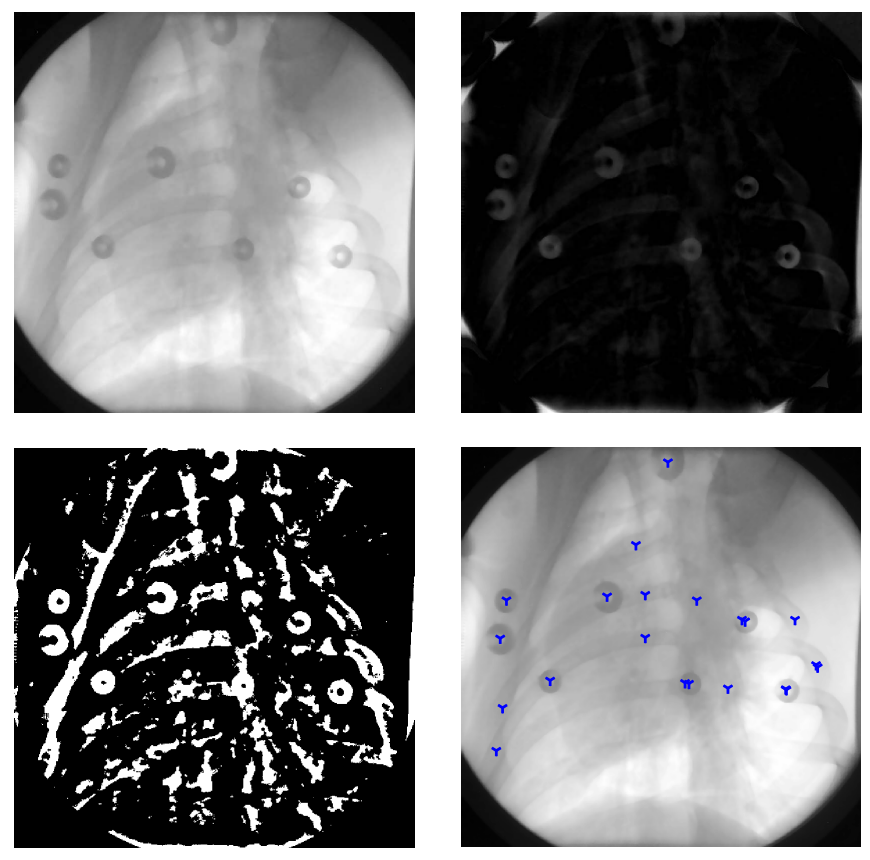

Fig. 3: The steps of the marker localization algorithm. (a) shows the input image, (b) the highlighting of marker regions using blackhat. (c) is the binarized image and (d) the resulting marker locations overlayed on the input image.

markers close to the detector appear smaller than the markers close to the X-ray source. This is an effect of perspective projection. Template sizes of $19.5 \mathrm{~mm}$ and $26.5 \mathrm{~mm}$ on the detector are used.

Template matching is performed using normalized crosscorrelation [14]. Marker positions are detected as local maxima with a correlation value larger than the empirically found 0.4. Fig. 3 shows the steps of the localization algorithm and the resulting marker locations after correlation with both templates. The false positives are not problematic, as they are filtered in the $3-\mathrm{D}$ reconstruction.

\section{Reconstruction of 3-D Marker Position in X-Ray}

After the 2-D localization, the marker positions are known in a set of X-ray images. The acquisition geometry of the images is given by the X-ray scanner. It is unknown which detected 2-D marker position belongs to which 3-D marker in different images. This is commonly known as the correspondence problem. The correspondence problem can be avoided if the 3-D positions are calculated directly with backprojection. A similar approach is used to reconstruct a volume from a number of projections, for example in computed tomography [15]. In a discrete volume, the rays through the camera centers and the 2-D marker positions are summed up. The 3-D marker positions appear as local maxima in the volume.

In 3-D reconstruction, the voxels are filled successively by summing the contribution from each image. This would be inefficient in this case as only the marker centers contribute to the volume and not every pixel in the image. Most of the voxels have zero intensity. For this sparse problem, the volume can be filled quickly with ray-driven backprojection [16], [12]. In ray-driven backprojection, the 2-D marker centers are iteratively projected back on the volume, implying that only non-zero voxels have to be processed. An image of a generated volume is displayed in Fig. 4.

The algorithm to detect the maxima is simple. First, the global maximum of the volume is found. Second, the neighborhood of 26 voxels of the maximum is masked for the search for the next marker. This neighborhood also has high values, because a lot of rays that belong to the adjacent maximum must pass through it. As the markers have a certain size and are attached to the patient with a certain distance from each other, the next marker cannot be in this area. In addition, the rays that go through the maximum are removed from the volume. This avoids coincidental intersections with these rays that might be misinterpreted as markers. This is achieved by storing the voxels for each ray that it passes through, and searching for all rays that pass through the maximum voxel and subtracting their contributions from the volume.

The volume is centered at the isocenter of the rotation of the $\mathrm{X}$-ray device. A sufficient size for the volume is 256 voxels in each dimension with a resolution of $2 \mathrm{~mm}$, thus covering a range of $51.2 \mathrm{~cm}$ in each direction. The low resolution is not a limitation for the accuracy of the system. The 3-D marker position can be refined by projecting the rough 3-D position found in the volume into the X-ray images. Then, the closest 2-D marker position to the projection of the markers in each image can be found and used for triangulation. In this context, the backprojection method is a way to establish correspondence between the markers.

After the correspondence is established, the 3-D points are computed in closed form from the 2-D points using linear triangulation [17]. Triangulation is heavily influenced by outliers, i.e. a single point that was localized incorrectly in 2-D directly influences the 3-D position. To prevent this, an outlier detection scheme is used. After triangulation with the complete set of 2-D points, the squared distances of each points ray to the estimated 3-D point is calculated. The maximum distance $d_{\text {max }} \in \mathbb{R}^{+}$is selected and analyzed further. If $d_{\max }>\alpha+\beta \mu$, with $\mu \in \mathbb{R}$ being the mean square distance of the non-maximum points and constants $\alpha, \beta \in \mathbb{R}$, the 2 -D point corresponding to $d_{\max }$ is removed from the set of points used for triangulation. The parameters were empirically set to $\alpha=1, \beta=2$. If $d_{\max } \leq \alpha+\beta \mu$, all the points contribute approximately equally to the $3-\mathrm{D}$ error and none is an outlier. This process is repeated until no more outliers are present or until only two points are left.

\section{Bundle Adjustment}

Now, the 2-D locations of the markers and an estimate of the 3-D position of the markers from the X-ray images are known. This section describes how the estimate of the 3-D position and the projection geometry of the X-ray images can be refined.

The reconstruction of 3-D points from a set of corresponding 2-D points in different projections can be seen as a geometric parameter estimation problem, with the parameters 


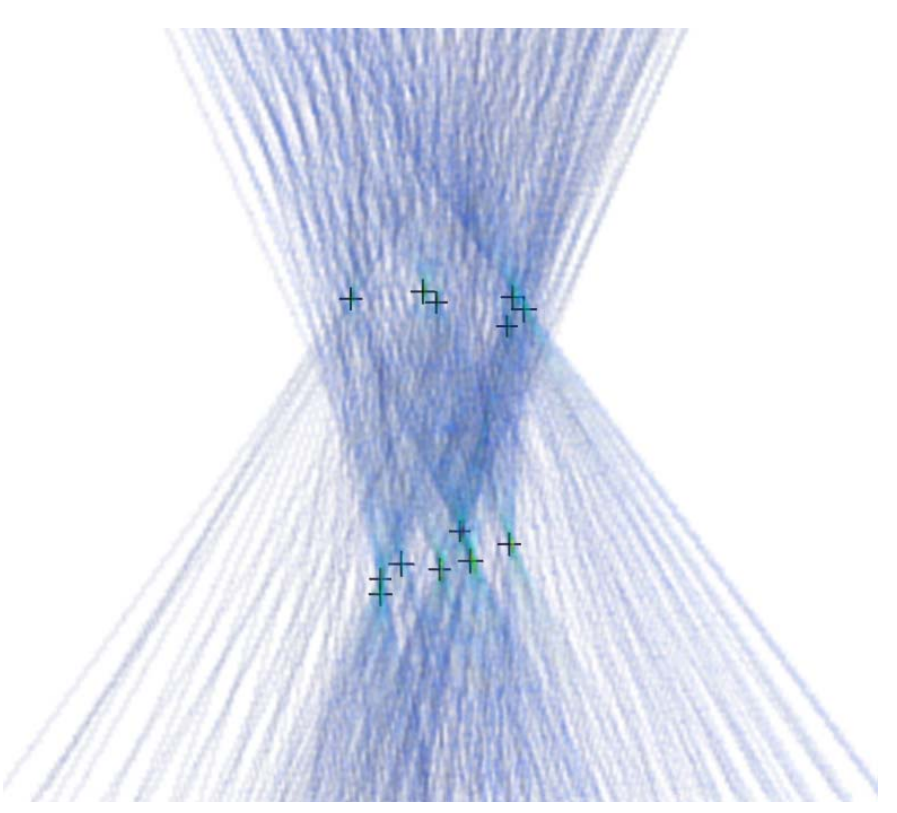

Fig. 4: The reconstructed volume used for finding the correspondence between projections. The black crosses are the detected 3-D markers. The volume is encoded with a colormap from blue for low values to red for high values.

being the coordinates of the 3-D points. The modification of bundle adjustment is to vary the projection geometry as well. The projection geometry involves internal and external camera parameters [18]. This is justified if the cameras are not calibrated. X-ray systems may be used without sufficient calibration in practice, as the calibration procedure has to be repeated regularly.

Bundle adjustment is a nonlinear optimization problem minimizing a geometric error. The name originates from the bundle of rays between the camera centers and the 3-D points that are adjusted to minimize the cost function [19]. A common choice for the cost function to be minimized is the mean square 2-D reprojection error $\epsilon_{B A} \in \mathbb{R}^{+}$:

$$
\epsilon_{B A}=\sum_{i, j}\left\|\mathbf{x}_{i j}-\hat{\mathbf{P}}_{j} \hat{\mathbf{X}}_{i}\right\|^{2} .
$$

The 2-D points $\mathbf{x}_{i, j} \in \mathbb{R}^{3}$ and the 3-D points $\hat{\mathbf{X}}_{i} \in \mathbb{R}^{4}$ are in homogeneous coordinates. The index $i$ runs over all 3-D points, $j$ over all projection images. Contrary to triangulation, the projection matrices $\hat{\mathbf{P}}_{j} \in \mathbb{R}^{3 \times 4}$ are also variable, which is indicated by the hat.

The optimization problem is solved using LevenbergMarquardt [20]. Bundle adjustment needs a good initialization of the 3-D points and the camera parameters to find the global optimum. All the methods for 3-D reconstruction presented so far are basically a way to find a good initialization.

The reconstruction problem in this project is rather small. There are only 5 to 20 views with fixed internal camera parameters and 10 to 14 markers. Problems of this size can be tackled with standard algorithms in acceptable time. In addition, outliers are already removed, so the least squares cost function can be used. The fact that the problem is small has disadvantages, too. There are only few 2-D measurements to constrain the solution. If the points and the external camera parameters are included in the optimization, the error is reduced to zero because the problem is underdetermined. Thus, it is necessary to keep the number of parameters as small as possible. It was decided to leave the points fixed and the external camera parameters variable. This creates $6 M$ degrees of freedom and reduces the projection error sufficiently. If only the points are variable, the projection error does not change much, which is a hint that $3 N$ degrees of freedom are not enough.

Bundle adjustment does not only improve the projections that are involved in the registration task. New images acquired later during the clinical procedure can be included, because bundle adjustment can be extended to these images if the markers are segmented.

\section{E. Rigid Registration}

The MR coordinate system and the X-ray coordinate system are not necessarily the same. The approximate relation is known, as the axis have a standardized meaning. Nevertheless, registration is necessary. The patient is transferred between MR and X-ray devices, and the table might not be positioned in exactly the same way, or the patient might have moved. Furthermore, manufacturing and calibration are not accurate enough to ensure that the coordinate systems are exactly as specified.

Computing the rigid registration between two 3-D point sets is a well known problem. It can be solved with the iterative closest point (ICP) algorithm introduced by Besl and McKay [21]. It is an iterative optimization algorithm that converges monotonically to the next local minimum of the mean square error

$$
\epsilon_{R}=\sum_{i=1}^{N}\left\|\mathbf{X}_{X F, i}-\hat{s} \hat{\mathbf{R}} \mathbf{X}_{M R, i}-\hat{\mathbf{t}}\right\|^{2} .
$$

The algorithm starts with an initial estimate for the rigid transform, including the scale $\hat{s} \in \mathbb{R}$, the rotation $\hat{\mathbf{R}} \in \mathbb{R}^{4 \times 4}$, and the translation $\hat{\mathbf{t}} \in \mathbb{R}^{4}$. Then, the correspondence is established by assigning each point $\mathbf{X}_{M R, i}$ to the closest point $\mathbf{X}_{X F, j}$ in the other set. For this purpose, the Euclidean distance between each pair of points in the Cartesian product of the two sets is computed. The points are assigned iteratively starting from the smallest distance and removing all the points that were already assigned from each of the sets. Having assigned all points, a closed-form algorithm for rigid registration with known point correspondence is used to get a new estimate for the rigid transform [22]. This is repeated iteratively until convergence. Termination criteria are insignificant change of error and maximum number of iterations.

In its basic form, the ICP algorithm is vulnerable to outliers in the dataset and converges only to a local minimum of the cost function. In the following, these two issues are addressed. Outliers occur frequently in this project, as neither of the localization algorithms works perfectly. This means that it is possible to have MR points that are no markers, MR points that are markers but do not have a corresponding XF point, XF points that are markers but have no corresponding MR point and XF points that are no markers. In addition, individual 
markers on the patient may have moved during the movement of the patient from the MR scanner to the X-ray device. These markers should also not be used for registration. The problem is that the least square error cost function of standard ICP is sensitive to outliers. To circumvent this, robust cost functions can be used in ICP [23], [24] or in a general-purpose optimization framework [25]. In this project, Trimmed ICP is used as it does not require significant modification of the algorithm [24]. Trimmed ICP does not minimize the least square error, but the least trimmed square error. In the step of computing the new rigid transform, instead of minimizing Eq. (2),

$$
\epsilon(\xi)=\sum_{i=1}^{\xi N}\left\|\mathbf{X}_{X F, i}-\hat{s} \hat{\mathbf{R}} \mathbf{X}_{M R, i}-\hat{\mathbf{t}}\right\|^{2}
$$

is minimized, where $\xi \leq 1$ is an overlap parameter. The points $\mathbf{X}_{X F, i}, \mathbf{X}_{M R, i}$ are sorted in order of increasing registration error. Thus, the registration is fitted only to the $\xi N$ points with the least square errors, and the other points are considered outliers. The overlap parameter $\xi$ is not known in advance. It can be determined automatically by scanning different overlaps and minimizing the objective function [24]

$$
\psi(\xi)=\frac{\epsilon(\xi)}{\xi^{1+\lambda}} .
$$

The objective function favors large overlaps if the error $e(\xi)$ is similar. $\lambda=2$ is a preset parameter that weights how much large overlap should be preferred over small error.

Two measures were implemented to increase the probability of finding the global maximum. Firstly, the algorithm is initialized with multiple transformations [21], [26]. This is implemented by randomly sampling the space of rotation matrices. Secondly, after the algorithm is stuck in a local minimum, the correspondence assignment of a random point is changed to the second nearest neighbor instead of the nearest neighbor. This is effective because the point sets are relatively small. The number of reassignments is limited by the maximum number of iterations allowed in ICP. Thus, some local minima can be avoided. Both globalization methods increase the runtime of the algorithm considerably. In order to maintain a good speed, ICP is terminated after a sufficiently good solution is found.

\section{EXPERIMENTS}

\section{A. Measures}

The 3-D X-ray marker localization algorithm and the 2-D localization accuracy were measured regarding the consistency of the locations in different projections. The 3-D localization error is the Euclidean distance of the ray connecting the camera center and the 2-D marker location on the detector to the estimated 3-D marker location. The root mean square (RMS) of this error is evaluated. In addition, the error was validated using leave-one-out cross validation (LOO), i.e. the 3-D location is computed with all markers except the one for which the error should be calculated. The 2-D localization error is measured as the Euclidean distance of the projection of
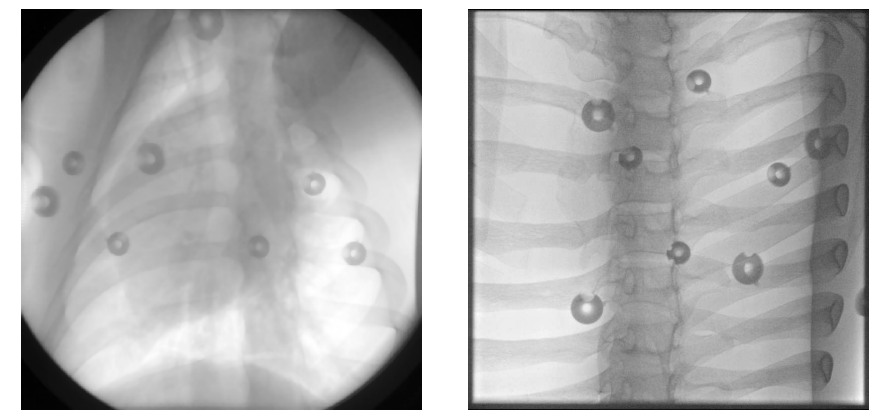

Fig. 5: Exemplary images acquired with the two different $X$ ray systems, (a) image intensifier system and (b) flat-panel detector system.

the estimated 3-D marker into an image to the corresponding detected 2-D marker location.

The measurement of the MR marker localization accuracy is more cumbersome, as there is no consistency or redundancy that can be exploited. The marker locations are extracted manually for three datasets, resulting in a total number of 40 markers. The 3-D localization error in MR is the Euclidean distance between the gold-standard annotation and the locations found by the algorithm. The RMS registration error is the 3-D Euclidean distance between corresponding markers from MR and X-ray. In addition, the error is computed with leave-one-out cross validation, i.e. the rigid transformation is computed, with the registration algorithm having as input all markers but the one of which the error should be calculated.

\section{B. Image Intensifier Data}

The X-ray fluoroscopy images are acquired with a singleplane XF system (Axiom Artis; Siemens AG, Healthcare Sector, Forchheim, Germany). This system has an image intensifier, which results in distorted images and a small field of view due to the circular shutter. The images have a size of $512 \times 512$ pixels at a resolution of $0.576 \times 0.576$, for an example see Fig. 5(a). The MR scanner is a $1.5 \mathrm{~T}$ system (Espree; Siemens AG, Healthcare Sector, Erlangen, Germany). The images are acquired with a $T_{1}$-weighted, gradient echo sequence and have a resolution of $2.3 \times 2.3 \times 1.6 \mathrm{~mm}$ and a size of $128 \times 128 \times 144$ voxels. There are seven datasets, each containing 20 projections evenly distributed over an angular range of $-30^{\circ}$ to $30^{\circ} \mathrm{LAO} / \mathrm{RAO}$-angle. The angle notation assumes a head first supine position of the patient. The RAO/LAO-angle denotes the rotation of a $\mathrm{C}$-arm related to a patients right/left side (right anterior oblique/left anterior oblique). The CRAN/CAUD-angle denotes the rotation towards a patients head (cranial) or feet (caudal). The errors are computed over all detected markers, using all images in which a marker is detected. The registration errors are computed over the best eight markers and over all detected markers. The use of only eight markers reflects the Trimmed ICP cost function, see Eq.(3), and simplifies comparison with the reference publication [12]. 


\section{Flat Panel Detector Data}

A current X-ray device with a flat panel detector is also tested (Artis zee; Siemens AG, Healthcare Sector, Forchheim, Germany). Flat panel detectors do not distort the images. The images have a size of $1920 \times 1920$ pixels at a resolution of $0.154 \times 0.154 \mathrm{~mm}^{2}$, see e.g. Fig. 5(b). The 14 datasets use between 11 and $21 \mathrm{X}$-ray images with an angular range of $-40^{\circ}$ to $40^{\circ} \mathrm{LAO} / \mathrm{RAO}$-angle and $-30^{\circ}$ to $30^{\circ} \mathrm{CRAN} / \mathrm{CAUD}$-angle. The 3-D MR is replaced with a 3-D C-arm CT. The automatic marker segmentation algorithm designed for MR cannot be applied, so the 3-D marker positions are extracted manually. Since no MR scanner is available, the 3-D MR localization error cannot be evaluated.

\section{Target Registration Error}

The clinical relevant error is the error of the overlaid MR information onto the live X-ray fluoroscopy images. Unfortunately, segmentation of anatomy in MR and the corresponding overlays are not available. Therefore, the situation is simulated by inserting a cube with small metallic beads as a target into the middle of the phantom. The beads can easily be detected in the X-ray images. The MR is again replaced with a C-arm $\mathrm{CT}$, where the markers for registration and the target beads are localized manually. The target overlay error is the 2D Euclidean distance between the projection of 3-D target locations and the corresponding 2-D target locations. The target registration error is the 3-D Euclidean distance between the 3-D target locations and the 3-D reconstruction of the segmented 2-D target locations. The experiment is conducted with the flat panel detector system on eight datasets with an angular range of $-40^{\circ}$ to $40^{\circ} \mathrm{LAO} / \mathrm{RAO}$-angle and $-30^{\circ}$ to $30^{\circ}$ CRAN/CAUD-angle containing between 11 and 21 images.

\section{E. Geometric Optimizations}

The C-arm X-ray device reports its acquisition geometry to the application. Acquisition geometry comprises e.g. LAO/RAO-angle, CRAN/CAUD-angle and 3-D position of the gantry. Due to production inaccuracies and aging, these values are not exact. These effects can be diminished by calibration, e.g. as described in [27]. Bundle adjustment has similar effects on the acquisition geometry. This experiment is designed to find out if calibration and bundle adjustment significantly improve the registration accuracy and to decide which method to use. To this end, all available datasets with different angular ranges and number of projections are processed with the registration algorithm without any geometric optimization, with calibration only, with bundle adjustment only and with both methods. The average localization error and the average registration error are measured.

\section{Results}

\section{A. Image Intensifier Data}

The 3-D MR localization error was $1.1 \mathrm{~mm}$ on average with a standard deviation of $0.5 \mathrm{~mm}$. All the 40 markers were detected correctly. In the X-ray images, the RMS 3-D

\begin{tabular}{clll}
\hline \multirow{2}{*}{ Evaluated error } & \multicolumn{3}{l}{ X-ray marker localization error $(\mathrm{mm})$} \\
\cline { 2 - 4 } & Mean \pm Std & Min & Max \\
\hline RMS & $0.32 \pm 0.35$ & 0.01 & 4.40 \\
LOO & $0.38 \pm 0.49$ & 0.01 & 6.02 \\
2-D & $0.40 \pm 0.52$ & 0.01 & 7.05 \\
\hline
\end{tabular}

TABLE I: Summary of localization results of 14 phantom measurements with flat panel detector data. The datasets contained between 11 and 21 projections.

\begin{tabular}{cllc}
\hline & \multicolumn{3}{l}{$\begin{array}{l}\text { Marker registration error for all } \\
\text { markers (mm) }\end{array}$} \\
\cline { 2 - 5 } Evaluated error & Mean \pm Std & Min & Max \\
\hline RMS & $1.50 \pm 1.78$ & 0.12 & 12.65 \\
LOO & $1.68 \pm 1.70$ & 0.16 & 12.63 \\
\hline
\end{tabular}

TABLE II: Summary of registration results of 14 phantom measurements with flat panel detector data. The datasets contained between 11 and 21 projections.

localization error is $0.79 \mathrm{~mm}$ on average, with a standard deviation of $0.54 \mathrm{~mm}$. The minimal error is close to $0.01 \mathrm{~mm}$, and the maximal error is $3.67 \mathrm{~mm}$. The LOO error is always a bit larger than the root mean square error. The mean 2-D localization error is $3.97 \mathrm{~mm}$.

The mean of the registration errors over the best eight markers is $3.04 \mathrm{~mm}$ in the RMS case and $7.25 \mathrm{~mm}$ in the LOO case. The error only slightly increased to $7.57 \mathrm{~mm}$ for the LOO case when computing it for all the markers, while it increases to $6.92 \mathrm{~mm}$ for the RMS case. The maximal RMS error is $72.21 \mathrm{~mm}$ and the maximal LOO error is $41.02 \mathrm{~mm}$, which reflects an incorrectly calculated 3-D X-ray marker position due to inexact localization.

\section{B. Flat Panel Detector Data}

The flat panel detector results are presented in TABLE I and TABLE II. The RMS X-ray localization error is on average $0.32 \mathrm{~mm}$ with a standard deviation of $0.35 \mathrm{~mm}$. The LOO X-ray localization error is $0.38 \mathrm{~mm}$ with a standard deviation of $0.49 \mathrm{~mm}$, which is less than half the size of the image intensifier experiments. The reduction of the 2-D Xray localization error in the flat panel detector experiments to $0.4 \mathrm{~mm}$ on average is attributed to the higher resolution of the images. The error in pixels is comparable to the image intensifier experiments.

The improvements are even greater for the registration error. For the best eight markers, the mean RMS error is $0.75 \mathrm{~mm}$ and the mean LOO error is $1.64 \mathrm{~mm}$. For all markers, the errors increase only slightly to $1.5 \mathrm{~mm}$ and $1.78 \mathrm{~mm}$, respectively. The maximal errors are again large compared to the mean, e.g. the maximal RMS error of $12.65 \mathrm{~mm}$ is eight times higher than the mean error.

\section{Target Registration Error}

The target overlay error is $1.1 \mathrm{~mm}$ on average with a standard deviation of $0.67 \mathrm{~mm}$. For better judgment of the magnitude of this error, which equals approximately 7 pixels, 


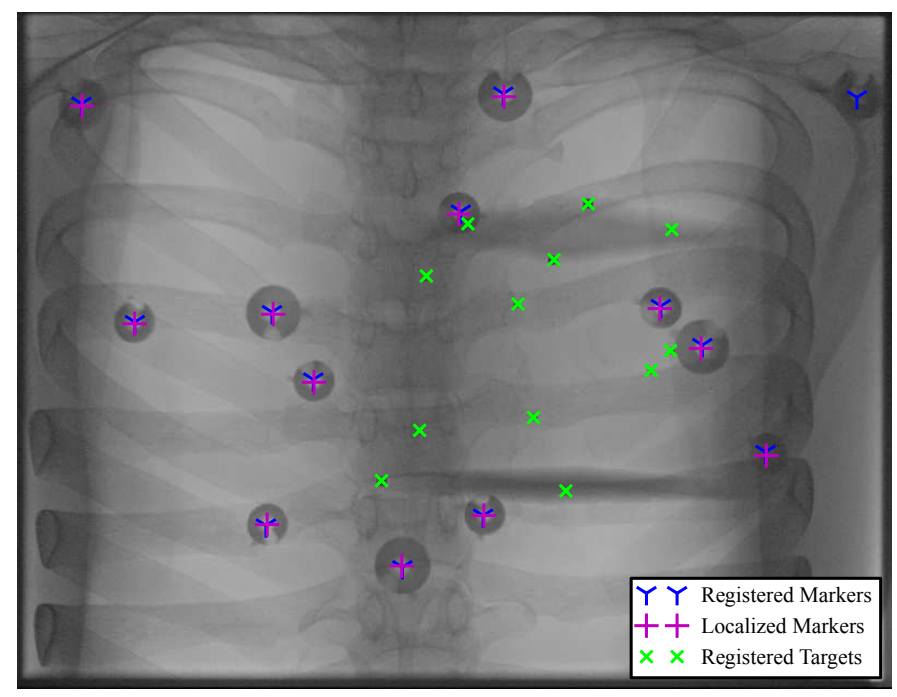

Fig. 6: Flat panel detector image showing the magnitude of segmentation, registration and target overlay errors.

the overlaid targets are presented in Fig. 6. The mean RMS target registration error is $1.4 \mathrm{~mm}$ with a standard deviation of $3.5 \mathrm{~mm}$. This is in the same range as the RMS marker registration error, compare TABLE II.

\section{Bundle Adjustment}

The average 3-D registration error is insusceptible to bundle adjustment and calibration. In fact, calibration slightly increases the median 3-D registration error from $0.95 \mathrm{~mm}$ to $1.01 \mathrm{~mm}$. The target registration error is slightly improved by bundle adjustment. This means that registration is better even for points that are not included in bundle adjustment, see Fig. 7 for different datasets. The influence on the localization error is more significant. As expected, the median 3-D localization error is highest when no optimization is performed, namely $0.34 \mathrm{~mm}$. Calibration reduces the 3 -D localization error to $0.29 \mathrm{~mm}$. Bundle adjustment alone reduces the error to $0.27 \mathrm{~mm}$, which is even better than calibration. If calibration and bundle adjustment are used together, the median 3-D localization error is $0.26 \mathrm{~mm}$ and thus similar to using only bundle adjustment. The 2-D X-ray localization error is also improved by bundle adjustment. The 2-D localization without bundle adjustment is $0.6 \pm 0.8 \mathrm{~mm}$. Bundle adjustment is able to reduce it to $0.4 \pm 0.5 \mathrm{~mm}$. Fig. 8 shows the improvements of bundle adjustment for several datasets.

\section{Discussion AND CONCLUSIONS}

The task of computing a registration between an MR volume dataset and fluoroscopic X-ray images is performed successfully for all available datasets.

The MR localization error is about $1.1 \mathrm{~mm}$, but the resolution of the MR scanner is larger than or equal to $1.6 \mathrm{~mm}$. As a consequence, the MR localization algorithm has subpixel accuracy. Additionally, all the markers are detected correctly in the analyzed datasets. Thus, the MR localization algorithm is robust and accurate. The accuracy of the XF

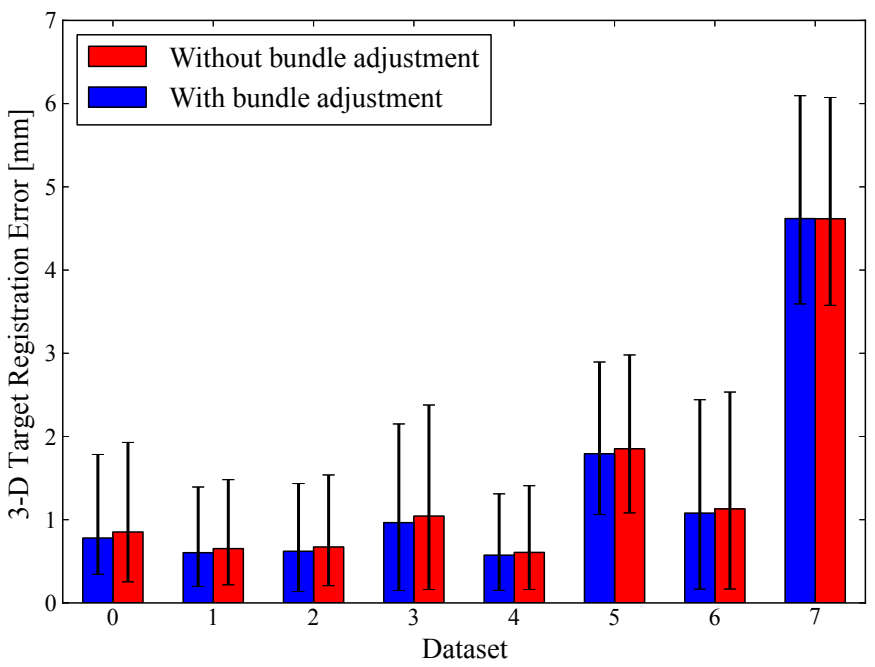

Fig. 7: The target registration error is improved slightly if bundle adjustment is used (blue bars). The results are shown for image intensifier data and they are consistent for all datasets.

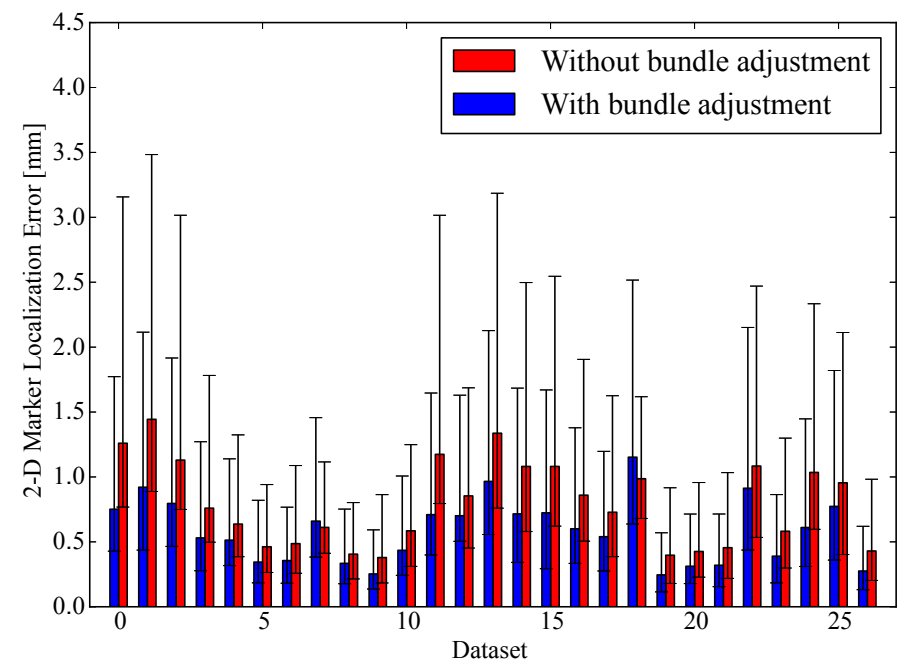

Fig. 8: Bundle adjustment (blue bars) reduces the the marker localization error. 27 datasets acquired with a flat panel detector system are evaluated.

localization method must be considered separately on the flat panel detector system and the image intensifier system. The image intensifier images are distorted and the resolution is low. In addition, the algorithm is designed to be used on a flat panel detector system. The relatively high root mean square error of $0.79 \mathrm{~mm}$ on average is therefore acceptable. The same error reduced to $0.38 \mathrm{~mm}$ if a flat panel detector system is used. The template matching algorithm benefits from higher resolutions, because the 2-D positions are detected only in integer pixel units. The registration error benefits even more from the newer system. Here, the mean RMS error for the best eight markers reduced from $3.04 \mathrm{~mm}$ to $0.75 \mathrm{~mm}$. Both registration and localization errors are higher than in the literature [12], where a localization error of $0.32 \mathrm{~mm}$ and a registration error of $1.16 \mathrm{~mm}$ were reported, using an image intensifier system but 
comparable or lower than the reference paper using a flat panel detector system. Therefore, the algorithm applied on a flat panel detector system is sufficiently accurate for ventricular septal defects and electrophysiology procedures as well. In [12], a registration error of less than $5 \mathrm{~mm}$ is said to be required for both procedures, which is higher than the average target registration error of $1.4 \mathrm{~mm}$ that was achieved by our proposed method.

Bundle adjustment and calibration are both suited to reduce projection errors, but they have limited influence on the registration accuracy. The effects of bundle adjustment are larger than the effects of calibration. To benefit from that, bundle adjustment should be applied during the intervention if the X-ray projection angles are not already included in the registration protocol. During an intervention the markers are still attached and thus can be detected using the same algorithms. These detected markers can serve as the input to a small bundle adjustment computation together with the previously computed 3-D points. To conclude, it is not necessary to use bundle adjustment or calibration to improve the computation of the rigid registration transform, but it can be useful during the procedure. Further testing in a clinical environment is necessary.

The execution of the program on a flat panel detector data set with 11 projections typically took $220 \mathrm{sec}$. The execution times were acquired on a Intel i5-2400 Dual Core CPU with 3.1 GHz and $4 \mathrm{~GB}$ RAM running on Windows 7 64bit. Most of the time is consumed in the 2-D X-ray marker localization algorithm, namely $78 \mathrm{~s}$ for the initial localization and $133 \mathrm{~s}$ for the localization refinement. The total execution time is still reasonable, as the physician is occupied with tasks that do not need the overlay in the beginning of the procedure.

\section{ACKNOWLEDGMENT}

We want to thank Anthony Faranesh and Robert Lederman from the Translational Medicine Branch of the Division of Intramural Research at the National Heart, Lung and Blood Institute in the National Institutes of Health, Bethesda, Maryland, USA for providing clinical data and support.

\section{REFERENCES}

[1] J. Sra, G. Narayan, D. Krum, A. Malloy, R. Cooley, A. Bhatia, A. Dhala, Z. Blanck, V. Nangia, and M. Akhtar, "Computed TomographyFluoroscopy Image Integration-Guided Catheter Ablation of Atrial Fibrillation," Journal of Cardiovascular Electrophysiology, vol. 18, no. 4, pp. 409-414, 2007.

[2] K. Rhode, D. Hill, P. Edwards, J. Hipwell, D. Rueckert, G. SanchezOrtiz, S. Hegde, V. Rahunathan, and R. Razavi, "Registration and Tracking to Integrate X-Ray and MR Images in an XMR Facility," IEEE Trans. Med. Imag., vol. 22, no. 11, pp. 1369-1378, 2003.

[3] A. Brost, R. Liao, J. Hornegger, and N. Strobel, "Model-Based Registration for Motion Compensation during EP Ablation Procedures," in Workshop on Biomedical Image Registration, Luebeck, Germany, ser. Lecture Notes in Computer Science, B. Fischer, B. Dawant, and C. Lorenz, Eds., vol. 6204. Springer, July 2010, pp. 234-245.

[4] R. de Silva, L. Gutiérrez, A. Raval, E. McVeigh, C. Ozturk, and R. Lederman, "X-Ray Fused with Magnetic Resonance Imaging (XFM) to Target Endomyocardial Injections," Circulation, vol. 114, no. 22, pp. 2342-2350, 2006.

[5] K. Ratnayaka, V. Raman, A. Faranesh, M. Sonmez, J. Kim, L. Gutiérrez, C. Ozturk, E. McVeigh, M. Slack, and R. Lederman, "Antegrade Percutaneous Closure of Membranous Ventricular Septal Defect using X-ray Fused with Magnetic Resonance Imaging," JACC Interventions, vol. 2 , no. 3 , p. $224,2009$.
[6] A. Krishnaswamy, E. Tuzcu, and S. Kapadia, "Three-dimensional Computed Tomography in the Cardiac Catheterization Laboratory," Catheterization and Cardiovascular Interventions, vol. 77, no. 6, pp. 860-865, 2011.

[7] D. Tomazevic, B. Likar, T. Slivnik, and F. Pernus, "3-D/2-D Registration of CT and MR to X-Ray Images," IEEE Trans. Med. Imag., vol. 22, no. 11, pp. 1407-1416, 2003.

[8] A. Gueziec, P. Kazanzides, B. Williamson, and R. Taylor, "AnatomyBased Registration of CT-Scan and Intraoperative X-Ray Images for Guiding a Surgical Robot," IEEE Trans. Med. Imag., vol. 17, no. 5, pp. 715-728, 1998

[9] T. Rohlfing and C. Maurer, "A Novel Image Similarity Measure for Registration of 3-D MR Images and X-ray Projection Images," in Medical Image Computing and Computer-Assisted Intervention - MICCAI 2002, Proceedings of the 5th International Conference, Tokyo, Japan, Part II, ser. Lecture Notes in Computer Science, T. Dohi and R. Kikinis, Eds., vol. 2489. Springer, September 2002, pp. 469-476.

[10] G. Penney, J. Weese, J. Little, P. Desmedt, D. Hill et al., "A Comparison of Similarity Measures for Use in 2-D-3-D Medical Image Registration," IEEE Trans. Med. Imag., vol. 17, no. 4, pp. 586-595, 1998.

[11] L. Zollei, E. Grimson, A. Norbash, and W. Wells, "2D-3D rigid registration of X-ray fluoroscopy and CT images using mutual information and sparsely sampled histogram estimators," in Proceedings of the 2001 IEEE Computer Society Conference on Computer Vision and Pattern Recognition - CVPR 2001, Kauai, HI, USA, vol. 2. IEEE, December 2001, pp. II-696 - II-703.

[12] A. George, M. Sonmez, R. Lederman, and A. Faranesh, "Robust Automatic Rigid Registration of MRI and X-Ray using External Fiducial Markers for XFM-Guided Interventional Procedures," Medical Physics, vol. 38, no. 1, pp. 125-141, January 2011.

[13] B. Brinkmann, A. Manduca, and R. Robb, "Optimized Homomorphic Unsharp Masking for MR Grayscale Inhomogeneity Correction,' IEEE Trans. Med. Imag., vol. 17, no. 2, pp. 161-171, 1998.

[14] G. Bradski and A. Kaehler, Learning OpenCV: Computer Vision with the OpenCV Library. O'Reilly Media, 2008.

[15] M. Slaney and A. Kak, Principles of Computerized Tomographic Imaging. New York: IEEE, 1988.

[16] F. Jacobs, E. Sundermann, B. De Sutter, M. Christiaens, and I. Lemahieu, "A Fast Algorithm to Calculate the Exact Radiological Path Through a Pixel or Voxel Space," Journal of Computing and Information Technology, vol. 6, no. 1, pp. 89-94, 1998.

[17] R. Hartley and P. Sturm, "Triangulation," Computer Vision and Image Understanding, vol. 68, no. 2, pp. 146-157, 1997.

[18] R. Hartley and A. Zisserman, Multiple View Geometry in Computer Vision, 2nd ed. Cambridge University Press, 2003.

[19] B. Triggs, P. McLauchlan, R. Hartley, and A. Fitzgibbon, "Bundle Adjustment - a Modern Synthesis," in Vision Algorithms: Theory and Practice, International Workshop on Vision Algorithms, held during ICCV 1999, Corfu, Greece, ser. Lecture Notes in Computer Science, B. Triggs, A. Zisserman, and R. Szeliski, Eds., vol. 1883. Springer, 2000, pp. 153-177.

[20] C. Kelley, Iterative Methods for Optimization. SIAM, 1999, vol. 18.

[21] P. Besl and N. McKay, "A Method for Registration of 3-D Shapes," IEEE Trans. Pattern Anal. Mach. Intell., vol. 14, no. 2, pp. 239-256, 1992.

[22] K. Arun, T. Huang, and S. Blostein, "Least-Squares Fitting of two 3-D Point Sets," IEEE Trans. Pattern Anal. Mach. Intell., vol. 9, no. 5, pp. 698-700, 1987

[23] E. Trucco, A. Fusiello, and V. Roberto, "Robust Motion and Correspondence of Noisy 3-D Point Sets with Missing Data," Pattern Recognition Letters, vol. 20, no. 9, pp. 889-898, 1999.

[24] D. Chetverikov, D. Svirko, D. Stepanov, and P. Krsek, "The Trimmed Iterative Closest Point Algorithm," in Proceedings of the 16th International Conference on Pattern Recognition, Quebec, Canada, R. Kasturi, D. Laurendau, and C. Suen, Eds., vol. 3. IEEE, August 2002, pp. 545-548.

[25] A. Fitzgibbon, "Robust Registration of 2D and 3D Point Sets," Image and Vision Computing, vol. 21, no. 13-14, pp. 1145-1153, 2003.

[26] B. Horn, "Relative Orientation," International Journal of Computer Vision, vol. 4, no. 1, pp. 59-78, 1990.

[27] A. Rougee, C. Picard, Y. Trousset, and C. Ponchut, "Geometrical Calibration for 3D X-Ray Imaging," in Medical Imaging 1993: Image Capture, Formatting, and Display, Newport Beach, CA, USA, Y. Kim, Ed., vol. 1897. SPIE, June 1993, pp. 161-169. [Online]. Available: http://link.aip.org/link/?PSI/1897/161/1 